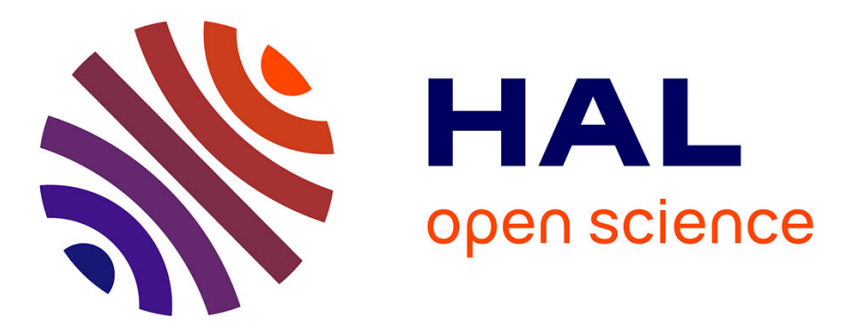

\title{
Leaderless synchronization of heterogeneous oscillators by adaptively learning the group model
}

\author{
Simone Baldi, Paolo Frasca
}

\section{To cite this version:}

Simone Baldi, Paolo Frasca. Leaderless synchronization of heterogeneous oscillators by adaptively learning the group model. IEEE Transactions on Automatic Control, 2020, 65 (1), pp.412-418. 10.1109/TAC.2019.2914664 . hal-02334955

\section{HAL Id: hal-02334955 https://hal.science/hal-02334955}

Submitted on 28 Oct 2019

HAL is a multi-disciplinary open access archive for the deposit and dissemination of scientific research documents, whether they are published or not. The documents may come from teaching and research institutions in France or abroad, or from public or private research centers.
L'archive ouverte pluridisciplinaire HAL, est destinée au dépôt et à la diffusion de documents scientifiques de niveau recherche, publiés ou non, émanant des établissements d'enseignement et de recherche français ou étrangers, des laboratoires publics ou privés. 


\title{
Leaderless synchronization of heterogeneous oscillators by adaptively learning the group model
}

\author{
Simone Baldi and Paolo Frasca
}

\begin{abstract}
This note addresses the problem of leaderless synchronization in a network of linear heterogeneous oscillators. It is well known that a synchronizing controller can be constructed when a common reference model is available to (some of) the agents. In this note, we show that synchronization can also be achieved without any access to such reference, by letting the agents cooperatively learn a suitable common model, which we refer to as group model. We show that there exists a group model that has the same structure as the oscillators and that the agents can learn its parameters and synchronize to it, by using a combination of consensus dynamics and adaptive regulation. This learning is even possible if the agents do not know their own dynamics, by using adaptive state observers. The distinguishing feature of this approach is making the agents collectively selforganize to their natural group model, instead of making them synchronize to an external reference.
\end{abstract}

Index Terms-Adaptive synchronization, heterogeneous oscillators, unknown dynamics.

\section{INTRODUCTION}

A $\mathrm{N}$ important problem in cooperative control is to achieve a common behavior for the entire network in a distributed way (i.e. using local information): this is the so-called synchronization problem [1]-[3], which is sometimes referred to as the consensus problem when the behaviour to be achieved is a constant value [4]-[6]. While static diffusive couplings between the agents are sufficient to ensure synchronization between homogeneous agents [7], synchronizing heterogeneous agents is essentially harder and static couplings are not sufficient [8]. Crucially, the existence of a common reference model is necessary for linear output synchronization. If this common reference model is available to all agents, synchronization can be achieved under mild connectivity assumptions [1], [9]. In some variations of the synchronization problem, the agents can also synchronize to a leader exosystem, provided at least some of the agents can access the exosystem signal. The idea behind this approach is that the agents that are not connected to the exosystem generating the reference signal will construct an observation of such signal in a distributed way, by coupling the so-called regulator equations with a distributed observer [10]-[12]. A common assumption to all these works is that all the agents know the common dynamical model to which they need to synchronize: this assumption can be quite restrictive and, recently, the authors of [13] have relaxed it by showing

S. Baldi is with Delft Center for Systems and Control, Delft University of Technology, Delft, The Netherlands and with School of Mathematics, Southeast University, Nanjing, China. E-mail: s.baldi@tudelft.nl

P. Frasca is with Univ. Grenoble Alpes, CNRS, Inria, Grenoble INP, GIPSALab, Grenoble, France. E-mail: paolo.frasca@gipsa-lab.fr

The authors would like to thank Claudio De Persis for insightful discussions that have lead to define the problems studied in this paper. that the common model can be known to some agents only, while being estimated in a distributed way by the others.

However, relevant questions remain open: what if none of the agents knows the common model? And pushing the boundary of uncertainty even further: what if the agents neither know their own dynamics? To the best of the authors' knowledge, the study of adaptive synchronization for heterogeneous uncertain systems is limited to special classes of systems/uncertainty, e.g. unknown control directions [14], unknown leader parameters [15], uncertain systems in output-feedback [16] or parametric strict-feedback [17] form. Leaderless consensus to a constant trajectory without resorting to any common model has been shown in classes of Euler-Lagrange [18] or passive systems [19, Chap. 8]. However, synchronization to more complex trajectories (e.g. periodic) would necessarily require a leader for which a desired trajectory is explicitly defined.

In this note we answer, for a class of linear agents that are harmonic oscillators, two questions: how to achieve synchronization when a common model is unknown to all agents? How to achieve synchronization when even the agent dynamics are unknown? None of the aforementioned approaches answers these two questions.

Our analysis focuses on harmonic oscillators, i.e. secondorder point-mass systems exhibiting periodic motion. These systems have recently attracted increasing attention, as some of the application fields include resonance phenomena, acoustic vibrations, electrical networks, motion coordination [20]-[24]. From a theory point of view, harmonic oscillators are also suitable to effectively using adaptive control tools, since they guarantee persistence of excitation. As compared to literature on nonlinear oscillators, e.g. limit-cycle or phase oscillators [25], [26], harmonic oscillators allow, via linear regulation theory, for necessary and sufficient conditions regarding the existence of a group model: in fact, being the group model a priori unknown, it is fundamental to study a setting whose solution is well posed, even in the presence of uncertainty. For example, in synchronization of nonlinear agents via nonlinear regulation theory [27], a solution may not exist if parametric uncertainty is too large.

\section{PROBLEM FORMULATION AND PRELIMINARIES}

We consider $n$ linear heterogeneous harmonic oscillators, indexed by $i \in\{1, \ldots, n\}$

$$
\dot{x}_{i}=\underbrace{\left[\begin{array}{cc}
0 & 1 \\
-\omega_{i}^{2} & 0
\end{array}\right]}_{A_{i}} x_{i}+\underbrace{\left[\begin{array}{c}
0 \\
1
\end{array}\right]}_{B_{i}=B} u_{i}, \quad y_{i}=\underbrace{\left[\begin{array}{ll}
0 & 1
\end{array}\right]}_{C_{i}=C} x_{i}
$$

where for $i \in\{1, \ldots, n\}$ we let $x_{i} \in \mathbb{R}^{2}$ be the state, $y_{i} \in \mathbb{R}$ be the measurable output, $u_{i} \in \mathbb{R}$ be the input, and $\omega_{i}>0$. Without 
loss of generality, the oscillators (1) have been taken in the chain-of-integrators canonical form. Note that the pairs $\left(A_{i}, B\right)$ and $\left(C, A_{i}\right)$ in (1) are stabilizable and detectable, respectively, by construction.

The oscillators in (1) can communicate with each other according to an undirected connected graph $(\mathscr{N}, \mathscr{E})$ with node set $\mathscr{N}=\{1, \ldots, n\}$ and edge set $\mathscr{E} \subset \mathscr{N} \times \mathscr{N}$ : its (weighted) adjacency matrix is $\mathscr{A}=\left[a_{i j}\right]$, which satisfies the relations $a_{i i}=0$ and $a_{i j}=a_{j i}>0$ if $(j, i) \in \mathscr{E}$; its (weighted) Laplacian matrix is $\mathscr{L}=\left[l_{i j}\right]$, which satisfies the relations $l_{i i}=\sum_{j=1}^{n} a_{j i}$ and $l_{i j}=-a_{j i}$ if $j \neq i$. A control strategy $u_{i}$ is said to be a distributed control strategy if it respects the communication flows described by the graph $(\mathscr{N}, \mathscr{E})$.

We let $v \in \mathbb{R}^{2}$ be the state of a group model to be found in the form

$$
\dot{v}=S v
$$

and let $e_{i} \in \mathbb{R}$ be the regulated output in the form

$$
e_{i}=y_{i}-\underbrace{\left[\begin{array}{ll}
0 & 1
\end{array}\right]}_{R_{i}=R} v .
$$

We are now ready to formulate the following two problems:

Problem 1 (Synchronization with unknown group model). Given the network (1), find a distributed controller $u_{i}$ such that all systems synchronize to an a priori unknown group model in the form (2), i.e. $\lim _{t \rightarrow \infty} e_{i}=0, \forall i \in\{1, \ldots, n\}$.

Problem 2 (Synchronization with unknown dynamics and group model). Given the network (1), find a distributed controller $u_{i}$ such that, without the knowledge of the system parameters in (1), all systems synchronize to an a priori unknown group model (2), i.e. $\lim _{t \rightarrow \infty} e_{i}=0, \forall i \in\{1, \ldots, n\}$.

The following Lemmas will be used for stability analysis.

Lemma 1 (Stability under decaying disturbances [13]). Consider the following system

$$
\dot{x}=F x+F_{1}(t) x+F_{2}(t)
$$

where $F \in \mathbb{R}^{n \times n}$ is Hurwitz, $F_{1}(t)$ and $F_{2}(t)$ are bounded and piecewise continuous for all $t \geq t_{0}$. If $\lim _{t \rightarrow \infty} F_{1}, F_{2}=0$ (exponentially), then $\lim _{t \rightarrow \infty} x=0$ (exponentially).

Lemma 2 (Synchronization under decaying disturbances). Consider the perturbed leaderless synchronization dynamics

$$
\dot{x}_{i}=S x_{i}+K \sum_{j=1}^{n} a_{i j}\left(x_{j}-x_{i}\right)+f_{i}(t) x_{i}
$$

where $x_{i} \in \mathbb{R}^{m}, S \in \mathbb{R}^{m \times m}$ is marginally stable, $K \in \mathbb{R}^{m \times m}$ is such that $\left(S-\lambda_{k} K\right)$ is Hurwitz for all non-zero eigenvalues $\lambda_{k}$ of the Laplacian $\mathscr{L}$, and $f_{i}(t)$ is bounded, continuously differentiable, and converges to zero exponentially. The definition of the error $\varepsilon_{i}=\sum_{j=1}^{n} a_{i j}\left(x_{j}-x_{i}\right)$ leads to the error dynamics

$$
\dot{\varepsilon}=\left[\left(I_{n} \otimes S\right)+(\mathscr{L} \otimes K)\right] \varepsilon+F(t) \varepsilon
$$

where $\varepsilon=\left[\begin{array}{lll}\varepsilon_{1}^{\prime} & \varepsilon_{2}^{\prime} \cdots \varepsilon_{n}^{\prime}\end{array}\right]^{\prime}=\left(\mathscr{L} \otimes I_{m}\right) x, x=\left[\begin{array}{ll}x_{1}^{\prime} & x_{2}^{\prime} \cdots x_{n}^{\prime}\end{array}\right]^{\prime}$, and $F(t)=\operatorname{diag}\left(f_{1} I_{m}, \cdots, f_{n} I_{m}\right)$. Then, $\lim _{t \rightarrow \infty} \varepsilon_{i}=0, i \in\{1, \ldots, n\}$.

Proof. This proof is provided because, although similar results have appeared in the leader-follower setting [13], we are not aware of a leaderless counterpart. From the explicit solution of (6), superposition of a marginally stable autonomous linear system and a time-varying autonomous system with exponentially decreasing state matrix, we know that $\varepsilon$ is bounded. For undirected and connected graphs, there exists a unitary matrix $\mathscr{U} \in \mathbb{R}^{n \times n}$ such that $\mathscr{U}^{\prime} \mathscr{L} \mathscr{U}=\operatorname{diag}\left(0, \lambda_{2}, \ldots, \lambda_{n}\right) \triangleq \Lambda$, where $\lambda_{k}, k=2, \ldots, n$, are the non-zero eigenvalues of the Laplacian matrix $\mathscr{L}$. Define the transformation $\varepsilon=\left(\mathscr{U} \otimes I_{m}\right) \bar{\varepsilon}$ with $\bar{\varepsilon}=\left[\bar{\varepsilon}_{1}^{\prime} \bar{\varepsilon}_{2}^{\prime} \ldots \bar{\varepsilon}_{n}^{\prime}\right]^{\prime}$, where it can be checked that $\bar{\varepsilon}_{1}=0$ [28]. Consider the positive definite Lyapunov function candidate

$$
V=\frac{1}{2} \varepsilon^{T}\left(I_{n} \otimes P\right) \varepsilon
$$

with $P$ a positive definite matrix to be chosen later. Using (6)

$$
\dot{V}=\varepsilon^{\prime}\left(I_{n} \otimes P\right)\left[\left[\left(I_{n} \otimes S\right)+(\mathscr{L} \otimes K)\right] \varepsilon+F(t) \varepsilon\right]
$$

where we have used the property $(A \otimes B)(C \otimes D)=A C \otimes B D$. Using the transformation $\varepsilon=\left(\mathscr{U} \otimes I_{m}\right) \bar{\varepsilon}$ we have

$$
\begin{aligned}
\dot{V} & =\bar{\varepsilon}^{\prime}\left[\left(I_{n} \otimes P S\right)+(\Lambda \otimes P K)\right] \bar{\varepsilon}+\varepsilon^{\prime}\left(I_{n} \otimes P\right) F \varepsilon \\
& =\sum_{i=2}^{n} \underbrace{\bar{\varepsilon}_{i}^{\prime}\left[P\left(S-\lambda_{k} K\right)+\left(S-\lambda_{k} K\right)^{\prime} P\right]}_{\dot{V}_{i}} \bar{\varepsilon}_{i}+\underbrace{\varepsilon^{\prime}\left(I_{n} \otimes P\right) F \varepsilon}_{\bar{F}} .
\end{aligned}
$$

Being $\left(S-\lambda_{k} K\right)$ Hurwitz for all non-zero eigenvalues $\lambda_{k}$ of the Laplacian $\mathscr{L}$, there exists a matrix $P$ such that $P\left(S-\lambda_{k} K\right)+$ $\left(S-\lambda_{k} K\right)^{\prime} P<0$ that is, $\dot{V}_{i}$ is negative semidefinite: also, $\bar{F}$ goes to zero exponentially (being $\varepsilon$ bounded). After integrating (7) we have $V(t) \leq V(0)+\Xi$, where $\Xi=\int_{0}^{t}|\bar{F}(\tau)| d \tau$ is finite since the exponentially decaying term is integrable. Therefore, $V(t)$ is bounded. Furthermore, we derive that $\dot{V}(t)$ is a uniformly continuous function of time because $\ddot{V}(t)$ is a bounded function of time. In fact

$$
\begin{aligned}
\ddot{V}= & 2 \bar{\varepsilon}^{\prime}\left[\left(I_{n} \otimes P S\right)+(\Lambda \otimes P K)\right] \\
& \cdot\left[\left[\left(I_{n} \otimes S\right)+(\Lambda \otimes K)\right] \bar{\varepsilon}+\left(\mathscr{U}^{\prime} \otimes I_{m}\right) F \varepsilon\right]+\dot{\bar{F}}
\end{aligned}
$$

where all variables are bounded. Barbalat's lemma [29, Lemma 3.2.6] implies that $\dot{V} \rightarrow 0$ as $t \rightarrow \infty$ and hence $\varepsilon \rightarrow 0$.

\section{SYNCHRONIZATION WITH UNKNOWN GROUP MODEL}

If a group model $S$ in (2) were known, a distributed control strategy could be constructed, provided there exist solutions $\left(\Pi_{i}, \Gamma_{i}\right), i \in\{1, \ldots, n\}$ such that the following equations, commonly referred to as the regulator equations, hold

$$
\left\{\begin{array}{l}
A_{i} \Pi_{i}+B \Gamma_{i}=\Pi_{i} S \\
C \Pi_{i}=R .
\end{array}\right.
$$

Then, synchronization would be guaranteed by the distributed control scheme [1, Theorem 5]

$$
\begin{aligned}
u_{i} & =-F_{i}\left(z_{i}-\Pi_{i} \zeta_{i}\right)+\Gamma_{i} \zeta_{i} \\
\dot{z}_{i} & =A_{i} z_{i}+B_{i} u_{i}+L_{i}\left(y_{i}-C z_{i}\right) \\
\dot{\zeta}_{i} & =S \zeta_{i}+K \sum_{j=1}^{n} a_{i j}\left(\zeta_{j}-\zeta_{i}\right)
\end{aligned}
$$

provided $K, F_{i}, L_{i}$ are chosen such that

$$
S-\lambda_{k} K \quad A_{i}-B F_{i} \quad A_{i}-L_{i} C
$$


are Hurwitz for all $i \in\{1, \ldots, n\}$ and all non-zero eigenvalues $\lambda_{k}$ of the Laplacian $\mathscr{L}$ associated to $\mathscr{A}$.

Remark 1 (On the controller stability conditions). The controller (10) comprises: a static observer-based controller (10a), a Luenberger observer (10b), and a distributed observer (10c) for reference generation: therefore, for every agent, the controller has four states. The Hurwitz properties of $A_{i}-B F_{i}$ and of $A_{i}-L_{i} C$ can be guaranteed by design in decentralized way. Instead, the Hurwitz condition of $S-\lambda_{k} K$ in principle requires some knowledge about the network. However, provided $S$ is in the form of a harmonic oscillator, any $K=\kappa I$ with $\kappa>0$ guarantees the condition, irrespective of the network topology. In what follows, we take $K=\kappa I$.

Since we are assuming that all individual models are harmonic oscillators, it is natural to seek a group model that is a harmonic oscillator as well, namely

$$
S=\left[\begin{array}{cc}
0 & 1 \\
-\beta & 0
\end{array}\right] \text {. }
$$

The following result ensures that a suitable harmonic oscillator is indeed a solution to the regulator equations.

Proposition 1 (Existence of a harmonic oscillator solution). A solution to (9) for dynamics (1) is given by

$$
\begin{aligned}
& S=\left[\begin{array}{cc}
0 & 1 \\
-\frac{1}{n} \sum_{\ell=1}^{n} \omega_{\ell}^{2} & 0
\end{array}\right], \quad \Pi_{i}=\Pi=I, \\
& \Gamma_{i}=\left[\frac{1}{n} \sum_{\ell=1}^{n}\left(\omega_{i}^{2}-\omega_{\ell}^{2}\right) \quad 0\right] .
\end{aligned}
$$

Proof. The regulator equations (9) take the form

$$
\left[\begin{array}{cc}
0 & 1 \\
-\omega_{i}^{2} & 0
\end{array}\right] \Pi_{i}+\left[\begin{array}{l}
0 \\
1
\end{array}\right] \Gamma_{i}=\Pi_{i} S, \quad\left[\begin{array}{ll}
0 & 1
\end{array}\right] \Pi_{i}=R .
$$

If we assume the group model to be an oscillator, then, without loss of generality, $\Gamma_{i}=\left[\begin{array}{ll}\gamma_{1_{i}} & \gamma_{2_{i}}\end{array}\right]$ and the first equation in (9) boils down to

$$
\begin{aligned}
\left(\Pi_{i}\right)_{11}=\left(\Pi_{i}\right)_{22} & \gamma_{1_{i}}=-\left(\Pi_{i}\right)_{11}\left(\beta-\omega_{i}^{2}\right) \\
\left(\Pi_{i}\right)_{21}=-\beta\left(\Pi_{i}\right)_{12} & \gamma_{2_{i}}=-\left(\Pi_{i}\right)_{12}\left(\beta-\omega_{i}^{2}\right) .
\end{aligned}
$$

If we choose $\Pi_{i}=I$ (from the second equation in (9)), then we obtain $\gamma_{2_{i}}=0$ and $\gamma_{1_{i}}=-\beta+\omega_{i}^{2}$. Let us now take $\beta=\frac{1}{n} \sum_{\ell=1}^{n} \omega_{\ell}^{2}$, i.e. the group model is the average of the frequencies available in the network: this leads us to $\gamma_{1_{i}}=\omega_{i}^{2}-\frac{1}{n} \sum_{\ell} \omega_{\ell}^{2}$. Therefore, the solution to (9) is (12).

Proposition 1 suggests that the mean of the squared frequencies of oscillation gives a legitimate group model $S$. However, such an $S$ is not directly known to the individual agents. For this reason, we propose to estimate $S$ in a distributed way via consensus dynamics. More precisely, we replace each individual copy of $S$ in (10c) by a local version

$$
S_{i}=\left[\begin{array}{cc}
0 & 1 \\
-\beta_{i} & 0
\end{array}\right]
$$

and update $\beta_{i}$ by the consensus dynamics

$$
\dot{\beta}_{i}=\sum_{j=1}^{n} a_{i j}\left(\beta_{j}-\beta_{i}\right), \quad \beta_{i}(0)=\omega_{i}^{2} .
$$

For an undirected connected graph, it is well known that (14) converges to $\beta$ in (12) [30, Thm. 2.2].

The solution to Problem 1 arises from combining the distributed scheme (10) with the consensus dynamics (14), as formalized by the following result.

Theorem 1 (Group controller). Consider the network of harmonic oscillators (1) communicating according to an undirected connected graph $(\mathscr{N}, \mathscr{E})$ : then, the following distributed control strategy

$$
\begin{aligned}
& u_{i}=-F_{i}\left(z_{i}-\zeta_{i}\right)+\left[\omega_{i}^{2}-\beta_{i} \quad 0\right] \zeta_{i} \\
& \dot{z}_{i}=A_{i} z_{i}+B u_{i}+L_{i}\left(y_{i}-C z_{i}\right), \quad z_{i}(0)=0 \\
& \dot{\zeta}_{i}=\left[\begin{array}{cc}
0 & 1 \\
-\beta_{i} & 0
\end{array}\right] \zeta_{i}+\kappa \sum_{j=1}^{n} a_{i j}\left(\zeta_{j}-\zeta_{i}\right), \quad \zeta_{i}(0)=0 \\
& \dot{\beta}_{i}=\sum_{j=1}^{n} a_{i j}\left(\beta_{j}-\beta_{i}\right), \quad \beta_{i}(0)=\omega_{i}^{2}
\end{aligned}
$$

achieves synchronization to the group model $S$ as in (12), provided the Hurwitz conditions in (11). In addition, we have $\lim _{t \rightarrow \infty} S_{i}=S, i \in\{1, \ldots, n\}$.

Proof. Let us define $\hat{\Gamma}_{i}=\left[\begin{array}{ll}\omega_{i}^{2}-\beta_{i} & 0\end{array}\right]$ to be an estimate of $\Gamma_{i}=\left[\begin{array}{ll}-\frac{\sum_{\ell} \omega_{\ell}^{2}}{n}+\omega_{i}^{2} & 0\end{array}\right]$. Also, let us define $F_{\eta_{i}}=\Gamma_{i}-F_{i}$, $\hat{F}_{\eta_{i}}=\hat{\Gamma}_{i}-F_{i}$ and $\tilde{F}_{\eta_{i}}=\Gamma_{i}-F_{i}-F_{\eta_{i}}$. Motivated by the manifold to which we want to converge, we define the coordinate change $\tilde{x}_{i}=x_{i}-v$ and $\tilde{z}_{i}=z_{i}-v$, where $v$ satisfies $\dot{v}=S v$. The dynamics of these two signals are

$$
\begin{aligned}
\dot{\tilde{x}}_{i} & =A_{i} x_{i}+B u_{i}-S v=A_{i} x_{i}+B u_{i}-A_{i} v-B \Gamma_{i} v \\
& =A_{i} \tilde{x}_{i}+B \tilde{u}_{i} \\
\dot{\tilde{z}}_{i} & =A_{i} \tilde{z}_{i}+B \tilde{u}_{i}+L_{i} C\left(\tilde{x}_{i}-\tilde{z}_{i}\right)
\end{aligned}
$$

where we have used the fact that $e_{i}=C x_{i}-C v=C \tilde{x}_{i}$, and we have defined $\tilde{u}_{i}=u_{i}-\Gamma_{i} v=-F_{i} \tilde{z}_{i}+\hat{F}_{\eta_{i}}\left(\zeta_{i}-v\right)+\tilde{F}_{\eta_{i}} v$. Therefore, it is convenient to write the following dynamics

$$
\begin{array}{r}
{\left[\begin{array}{c}
\dot{\tilde{x}}_{i} \\
\dot{\tilde{x}}_{i}-\dot{\tilde{z}}_{i}
\end{array}\right]=\left[\begin{array}{cc}
A_{i}-B F_{i} & B F_{i} \\
0 & A_{i}-L_{i} C
\end{array}\right]\left[\begin{array}{c}
\tilde{x}_{i} \\
\tilde{x}_{i}-\tilde{z}_{i}
\end{array}\right]} \\
+\left[\begin{array}{c}
B \\
0
\end{array}\right] \hat{F}_{\eta_{i}}\left(\zeta_{i}-v\right)+\left[\begin{array}{c}
B \\
0
\end{array}\right] \tilde{F}_{\eta_{i}} v
\end{array}
$$

Observe that from (15c) and defining $\bar{a}_{i j}=\kappa a_{i j}$ we obtain

$$
\dot{\zeta}_{i}=S \zeta_{i}+\sum_{j=1}^{n} \bar{a}_{i j}\left(\zeta_{j}-\zeta_{i}\right)+\left(S_{i}-S\right) \zeta_{i}
$$

The first two terms of the right-hand side constitute the synchronization dynamics of homogeneous oscillators, while the third one is a disturbance that converges to zero exponentially, because $\lim _{t \rightarrow \infty} \beta_{i}=\beta$ and $\lim _{t \rightarrow \infty} S_{i}=S$ exponentially for all $i \in\{1, \ldots, n\}$. Thus, Lemma 2 allows to conclude $\lim _{t \rightarrow \infty} \zeta_{i}-v=0, i \in\{1, \ldots, n\}$. Finally, the term $\tilde{F}_{\eta_{i}}$ goes to zero exponentially. By looking at (16), we notice that the Hurwitz properties of $A_{i}-B F_{i}$ and of $A_{i}-L_{i} C$ make the system matrix of (16) Hurwitz and, in addition, the system is driven by decaying disturbances: therefore, Lemma 1 guarantees that $\tilde{x}_{i}$ converges to zero, from which we obtain convergence of $e_{i}$ to zero. This concludes the proof. 
Remark 2 (Extension to chains-of-integrators). Even though this note focuses on harmonic oscillators, we can show that the proposed results directly extend to the wider class of systems in the chain-of-integrators canonical form, resulting in

$$
\frac{Y_{i}(s)}{U_{i}(s)}=\frac{1}{s^{m}+a_{1_{i}} s^{m-1}+\ldots+a_{m_{i}}} .
$$

For all systems in the form (17), a possible solution to (9) is

$$
\begin{aligned}
S & =\left[\begin{array}{cc}
0 & I_{m-1} \\
-\frac{1}{n} \sum_{i} a_{m_{i}} & \ldots-\frac{1}{n} \sum_{i} a_{1_{i}}
\end{array}\right], \quad \Pi_{i}=I \\
\Gamma_{i} & =\left[\begin{array}{lll}
\frac{1}{n} \sum_{\ell=1}^{n}\left(a_{m_{i}}-a_{m_{\ell}}\right) & \ldots & \frac{1}{n} \sum_{\ell=1}^{n}\left(a_{1_{i}}-a_{1_{\ell}}\right)
\end{array}\right] .
\end{aligned}
$$

The extension to Theorem 1 follows directly.

\section{SYNCHRONIZATION WITH UNKNOWN DYNAMICS AND GROUP MODEL}

The implementation of (15) requires the knowledge of the parameter $\omega_{i}$, i.e. of the agent dynamics. In the following, we would like to remove this assumption and endow the agents with the ability to estimate it. To this purpose, we propose the following adaptive state observer

$$
\begin{aligned}
\dot{z}_{i} & =\underbrace{\left[\begin{array}{cc}
0 & 1 \\
-\hat{\bar{\omega}}_{i} & 0
\end{array}\right]}_{\hat{A}_{i}} z_{i}+\underbrace{\left[\begin{array}{l}
0 \\
1
\end{array}\right]}_{B} u_{i}+\underbrace{\left[\begin{array}{l}
l_{1_{i}}(t) \\
l_{2_{i}}(t)
\end{array}\right]}_{L_{i}}\left(y_{i}-\hat{x}_{2_{i}}\right) \\
\hat{x}_{2_{i}} & =\left[\begin{array}{cc}
0 & 1
\end{array}\right] z_{i}
\end{aligned}
$$

where $\hat{\bar{\omega}}_{i}$ is the estimate of $\bar{\omega}_{i}=\omega_{i}^{2}$ and $L_{i}(t)$ must be such that $\hat{A}_{i}(t)-L_{i}(t) C$ is Hurwitz at every time instant.

In order to produce the estimate $\hat{\bar{\omega}}_{i}$, we derive the following parametric model by exploiting the chain-of-integrators form of the oscillators

$$
\underbrace{\frac{s^{2}}{s^{2}+\lambda_{1} s+\lambda_{2}} x_{2_{i}}-\frac{s}{s^{2}+\lambda_{1} s+\lambda_{2}}}_{\xi_{i}} u_{i}=\omega_{i}^{2} \underbrace{\frac{-1}{s^{2}+\lambda_{1} s+\lambda_{2}} x_{2_{i}}}_{\phi_{i}}
$$

where the state has been decomposed as $x_{i}=\left[\begin{array}{ll}x_{1_{i}} & x_{2_{i}}\end{array}\right]$, and $\lambda_{1}, \lambda_{2}>0$ are such that $s^{2}+\lambda_{1} s+\lambda_{2}$ is a Hurwitz polynomial. Similarly to [29, Sect. 4.2], we have used a Laplace streamlined notation to indicate the filtering of the signals $x_{2_{i}}$ and $u_{i}$. The parametric model (20) is a linear-inthe-parameter model for which the following gradient-based adaptation law [29, Sect. 5.3] can be designed

$$
\begin{aligned}
\dot{\hat{\bar{\omega}}}_{i} & =\operatorname{Proj}\left[\gamma\left(\xi_{i}-\hat{\bar{\omega}}_{i} \phi_{i}\right) \phi_{i}\right], \quad \hat{\bar{\omega}}_{i}(0)=\hat{\bar{\omega}}_{0_{i}}>0 \\
& = \begin{cases}\gamma\left(\xi_{i}-\hat{\bar{\omega}}_{i} \phi_{i}\right) \phi_{i} & \text { if } \hat{\bar{\omega}}_{i}<0 \text { or } \\
0 & \text { if } \hat{\bar{\omega}}_{i}=0 \text { and }\left(\xi_{i}-\hat{\bar{\omega}}_{i} \phi_{i}\right) \phi_{i} \geq 0\end{cases}
\end{aligned}
$$

where $\gamma>0$ is an adaptive gain, $\hat{\bar{\omega}}_{0_{i}}$ is an initial estimate of the squared frequency $\omega_{i}^{2}, \hat{\bar{\omega}}_{i}$ is the on-line estimate of $\bar{\omega}_{i}$ and $\operatorname{Proj}[\cdot]$ is the projection operator to keep $\hat{\omega}_{i}>0$. In addition, the following proposition gives another suitable group model (2) for the network.
Proposition 2 (Alternate harmonic oscillator solution). $A$ solution to (9) is given by

$$
\begin{aligned}
& S=\left[\begin{array}{ccc}
0 & 1 \\
-\frac{1}{n} \sum_{\ell=1}^{n} \hat{\bar{\omega}}_{0_{\ell}} & 0
\end{array}\right], \quad \Pi_{i}=\Pi=I, \\
& \Gamma_{i}=\left[\frac{1}{n} \sum_{\ell=1}^{n}\left(\hat{\bar{\omega}}_{0_{i}}-\bar{\omega}_{\ell}\right) \quad 0\right]
\end{aligned}
$$

Proof. The proof follows the same steps as Proposition 1. Notice that, differently from (12), the group model $S$ is now chosen to be the average of the initial estimates $\hat{\bar{\omega}}_{0_{i}}$ of the squared frequencies in the network. It is important to mention that, even in the presence of uncertain dynamics, the solution (22) is always well defined when $\hat{\bar{\omega}}_{0_{i}}, \bar{\omega}_{i}>0$, $i \in\{1, \ldots, n\}$.

The solution to Problem 2 arises from combining the distributed scheme (10) with the adaptive state observer (19) and adaptive law (21), as formalized by the following result.

Theorem 2 (Group controller with unknown dynamics). Consider the network of harmonic oscillators (1) interconnected according to an undirected connected graph $(\mathscr{N}, \mathscr{E})$ : then, the following distributed control strategy

$$
\begin{array}{ll}
u_{i}=-F_{i}\left(z_{i}-\zeta_{i}\right)+\left[\begin{array}{cr}
\hat{\bar{\omega}}_{i}-\beta_{i} & 0
\end{array}\right] \zeta_{i} & \\
\dot{z}_{i}=\hat{A}_{i} z_{i}+B u_{i}+L_{i}\left(y_{i}-C z_{i}\right), & z_{i}(0)=0 \\
\dot{\zeta}_{i}=\left[\begin{array}{cr}
0 & 1 \\
-\beta_{i} & 0
\end{array}\right] \zeta_{i}+\kappa \sum_{j=1}^{n} a_{i j}\left(\zeta_{j}-\zeta_{i}\right), & \zeta_{i}(0)=0 \\
\dot{\beta}_{i}=\sum_{j=1}^{n} a_{i j}\left(\beta_{j}-\beta_{i}\right), & \beta_{i}(0)=\hat{\bar{\omega}}_{0_{i}} \\
\dot{\bar{\omega}}_{i}=\operatorname{Proj}\left[\gamma\left(\xi_{i}-\hat{\bar{\omega}}_{i} \phi_{i}\right) \phi_{i}\right], & \hat{\bar{\omega}}_{i}(0)=\hat{\bar{\omega}}_{0_{i}},
\end{array}
$$

achieves synchronization to an a priori unknown group model $S$, with $S$ as in (22), provided $F_{i}, L_{i}$ (which can be time-varying) are chosen such that

$$
\hat{A}_{i}-B F_{i} \quad \hat{A}_{i}-L_{i} C
$$

are Hurwitz at every time instant. In addition, we have $\lim _{t \rightarrow \infty} S_{i}=S, i \in\{1, \ldots, n\}$, with $S$ as in (22).

Proof. Similarly to the derivation of Theorem 1, we can find the dynamics of $\tilde{x}_{i}=x_{i}-v$ and $\tilde{z}_{i}=z_{i}-v$, which are

$$
\begin{aligned}
& \dot{\tilde{x}}_{i}=A_{i} \tilde{x}_{i}+B \tilde{u}_{i} \\
& \dot{\tilde{z}}_{i}=\hat{A}_{i} \tilde{z}_{i}+B \tilde{u}_{i}+L_{i} C\left(\tilde{x}_{i}-\tilde{z}_{i}\right)+\tilde{A}_{i} v
\end{aligned}
$$

where $\tilde{A}_{i}=\hat{A}_{i}-A_{i}$ : also, we have used the fact that $e_{i}=C x_{i}-$ $C v=C \tilde{x}_{i}$, and we have defined $\tilde{u}_{i}=-F_{i} \tilde{z}_{i}+\hat{F}_{\eta_{i}}\left(\zeta_{i}-v\right)+\tilde{F}_{\eta_{i}} v$. This leads to the following dynamics

$$
\begin{aligned}
{\left[\begin{array}{c}
\dot{\tilde{x}}_{i} \\
\dot{\tilde{x}}_{i}-\dot{\tilde{z}}_{i}
\end{array}\right]=} & {\left[\begin{array}{cc}
\hat{A}_{i}-B F_{i} & B F_{i} \\
0 & \hat{A}_{i}-L_{i} C
\end{array}\right]\left[\begin{array}{c}
\tilde{x}_{i} \\
\tilde{x}_{i}-\tilde{z}_{i}
\end{array}\right] } \\
& +\left[\begin{array}{c}
-\tilde{A}_{i} \tilde{x}_{i}+B \hat{F}_{\eta_{i}}\left(\zeta_{i}-v\right)+B \tilde{F}_{\eta_{i}} v \\
-\tilde{A}_{i} x_{i}
\end{array}\right] .
\end{aligned}
$$

In addition, it is convenient to write the dynamics of $z_{i}-\zeta_{i}$

$$
\dot{z}_{i}-\dot{\zeta}_{i}=\left(\hat{A}_{i}-B F_{i}\right)\left(z_{i}-\zeta_{i}\right)+L_{i} C\left(x_{i}-z_{i}\right)-\kappa \sum_{j=1}^{n} a_{i j}\left(\zeta_{j}-\zeta_{i}\right) .
$$


By observing the terms on the right-hand side of (25), we have that $\lim _{t \rightarrow \infty} C\left(x_{i}-z_{i}\right)=0$ (because a well-known result of the adaptive observer (19) is that the output observation error $y_{i}-\hat{x}_{2_{i}}$ converges to zero for $t \rightarrow \infty$ [29, Thm. 5.3.1]) and $\lim _{t \rightarrow \infty} \sum_{j=1}^{n} a_{i j}\left(\zeta_{j}-\zeta_{i}\right)=0$ (by applying Lemma 2 as in the proof of Theorem 1). Therefore, being $\hat{A}_{i}-B F_{i}$ Hurwitz, we have that the system matrix of (25) is Hurwitz. Therefore, similarly to (4), we have stable dynamics driven by decaying disturbances. From Lemma 1 we obtain convergence to zero of the state of (25), i.e. $\lim _{t \rightarrow \infty} z_{i}-\zeta_{i}=0$. Now, by looking at (23), we have that $u_{i}$ is the sum of two terms: a vanishing one $\left(z_{i}-\zeta_{i}\right)$ and a sinusoidal one $\left(\zeta_{i}\right)$. It is well known that the state of a harmonic oscillator is persistently exciting: using standard properties on persistently exciting signals [29, Lemma 4.8.3], we have that $u_{i}$ is sufficiently rich of order 2 [29, Def. 5.2.1].

An adaptive observer with sufficiently rich inputs guarantees that the state observation error $x_{i}-z_{i}$ and the parameter error $\hat{\bar{\omega}}_{i}-\bar{\omega}_{i}$ converge to zero exponentially fast [29, Thm. 5.3.1]. The last convergence implies that the term $\tilde{A}_{i}$ in (24) also converges to zero exponentially fast. At this point we are in a similar situation as in Theorem 1, with an asymptotically stable system affected by decaying disturbances: therefore, Lemma 1 guarantees that $\tilde{x}_{i}$ converges to zero, from which we obtain convergence of $e_{i}$ to zero. This concludes the proof.

Remark 3 (Actual $\bar{\omega}_{i}=\omega_{i}^{2}$ and estimated $\hat{\bar{\omega}}_{i}$ ). The difference between the consensus dynamics in (23d) and those in (15d) is the initial condition, which reflects the a priori knowledge. In fact, in (15d) we can use the known $\omega_{i}^{2}$ as initial conditions, while in (23d), being the actual dynamics unknown, we can use only their initial estimates $\hat{\bar{\omega}}_{0_{i}}$. Despite unknown dynamics, a solution (22) is always guaranteed to exist.

\section{A. Average model as group model}

It must be noted that the solution to Problem 2 presented so far in this section does not guarantee the group model to be the average of the actual individuals models, as instead was the case in our solution to Problem 1 . In other words, $S$ in (12) (average of the actual individuals models) might be different from $S$ in (22) (average of the estimated individuals models). With the pursuit of bridging this gap, we now provide an extension to our framework (23) that is able to converge to a group model $S$, with $S$ as in (12), i.e. being the average of the actual individuals models. We propose the following adaptive controller

$$
\begin{array}{rlrl}
u_{i} & =-F_{i}\left(z_{i}-\zeta_{i}\right)+\left[\begin{array}{ll}
\hat{\bar{\omega}}_{i}-\beta_{i} & 0
\end{array}\right] \zeta_{i} & \\
\dot{z}_{i} & =\hat{A}_{i} z_{i}+B u_{i}+L_{i}\left(y_{i}-C z_{i}\right), & & z_{i}(0)=0 \\
\dot{\zeta}_{i} & =\left[\begin{array}{cr}
0 & 1 \\
-\beta_{i} & 0
\end{array}\right] \zeta_{i}+\kappa \sum_{j=1}^{n} a_{i j}\left(\zeta_{j}-\zeta_{i}\right), & \zeta_{i}(0)=0 \\
\dot{\beta}_{i} & =\dot{\hat{\bar{\omega}}}_{i}+\sum_{j=1}^{n} a_{i j}\left(\beta_{j}-\beta_{i}\right), & & \beta_{i}(0)=\hat{\bar{\omega}}_{0_{i}} \\
\dot{\hat{\bar{\omega}}}_{i} & =\operatorname{Proj}\left[-\gamma\left(R_{\omega} \hat{\bar{\omega}}_{i}+Q_{\omega}\right)\right], & \hat{\omega}_{i}(0)=\hat{\bar{\omega}}_{0_{i}} \\
\dot{R}_{\omega} & =-\mu R_{\omega}+\phi_{i}^{2}, & R_{\omega}(0)=0 \\
\dot{Q}_{\omega} & =-\mu Q_{\omega}+\phi_{i} \xi_{i}, & & Q_{\omega}(0)=0,
\end{array}
$$

with $\gamma, \mu>0$ being adaptive gains, and provided the Hurwitz conditions on $\hat{A}_{i}-B F_{i}$ and $\hat{A}_{i}-L_{i} C$. The essential differences with respect to (23) lie in the modified consensus (26d) and in the estimation scheme (26e)-(26g). The former exploits the availability of $\dot{\hat{\bar{\omega}}}_{i}$ in order for $\beta_{i}$ to converge to the actual average of squared frequencies, while the latter is a gradientbased estimation with integral cost [29, Thm. 4.3.3].

Remark 4 (Convergence proof for (26)). As compared to (23), the additional difficulty in the analysis of (26) lies in guaranteeing convergence of the consensus dynamics (26d) in the presence of the extra term $\dot{\overline{\hat{\omega}}}_{i}$ : for the particular case of gradient-based estimation with integral cost, it is well known that $\lim _{t \rightarrow \infty} \dot{\overline{\hat{\omega}}}_{i}=0$ irrespective of the input $u_{i}$ [29, Thm. 4.3.3] (a similar result holds for least square estimation with no forgetting factor [29, Thm. 4.3.4]). Therefore, in such special estimation algorithms, $\dot{\hat{\bar{\omega}}}_{i}$ acts as a vanishing disturbance on the consensus dynamics, which allows us to use Lemma 1 to guarantee $\lim _{t \rightarrow \infty} z_{i}-\zeta_{i}=0$ and therefore convergence to the state of a (persistently exciting) group model. The rest of the proof follows similar steps as for Theorem 2. For standard gradient-based estimation laws like the one in (23e), convergence of $\dot{\hat{\bar{\omega}}}_{i}$ to zero irrespective of $u_{i}$ cannot be proven: at most, one can prove that $\dot{\overline{\hat{\omega}}}_{i}$ is bounded and with finite energy [29, Thm. 4.3.2]. Therefore, it seems difficult to generalize (26) to any estimation algorithm.

Despite the difficulty in generalizing the algorithm (26), numerical experiments (cf. Sect. V) suggest that the combination of (26a)-(26d) and (23e) (i.e. the modified consensus with the standard gradient-based estimation) is able to converge to the actual average of squared frequencies.

\section{NUMERICAL EXAMPLES}

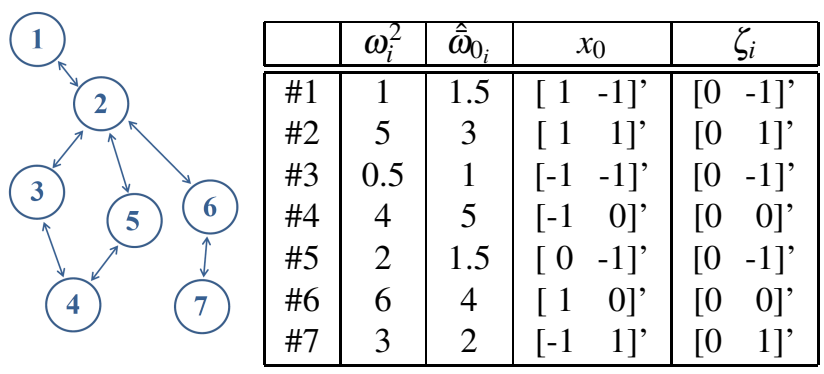

Fig. 1: The undirected communication graph

Simulations are carried out on the undirected graph shown in Fig. 1, where the table reports the squared frequencies $\omega_{i}^{2}$ of each oscillators and the initial estimates $\hat{\bar{\omega}}_{0_{i}}$ (the latter to be used for the case in which $\bar{\omega}_{i}=\omega_{i}^{2}$ is unknown). The other parameters are: $F_{i}$ are chosen such that $A_{i}-B F_{i}\left(\right.$ or $\left.\hat{A}_{i}-B F_{i}\right)$ have poles in -0.75 and $-1.5 ; L_{i}$ are chosen such that $A_{i}-L_{i} C$ (or $\hat{A}_{i}-L_{i} C$ ) have poles in -2.25 and $-4.5 ; \kappa=3$ for all $i$, $z_{i}(0)=0$ for all $i, \lambda_{1}=2, \lambda_{2}=1$ and $\gamma=100$.

On this set-up, we simulated the evolution of the controlled dynamics by using controllers (15), (23) and (26). In case $\omega_{i}^{2}$ are known, the outputs $y_{i}$ and inputs $u_{i}$ resulting from (15) are shown in Fig. 2: synchronization of the outputs to the same 

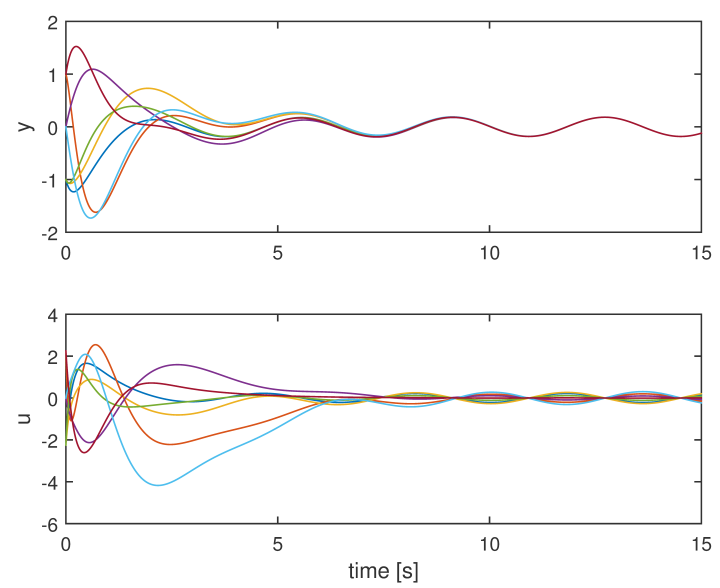

Fig. 2: Known $\omega_{i}^{2}$ : synchronizing outputs $y_{i}$ and inputs $u_{i}$.
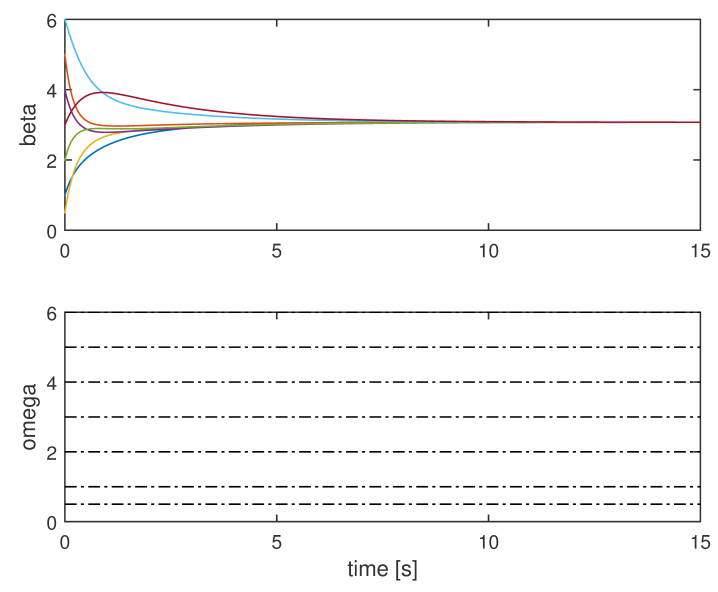

Fig. 3: Known $\omega_{i}^{2}$ : convergence of $\beta_{i}$.

frequency is achieved, whereas it can be noted that each agent has a different input in view of the heterogeneous dynamics. The frequency to which the systems converge is defined by the convergence of $\beta_{i}$, as shown in Fig. 3. In case $\omega_{i}^{2}$ are unknown, they cannot be used for control design and (23) is employed. The resulting outputs $y_{i}$ and inputs $u_{i}$ are shown in Fig. 4. The frequency to which all agents synchronize depends upon the consensus dynamics over $\hat{\bar{\omega}}_{0_{i}}$ as shown in Fig. 5: therefore the synchronizing frequency is not necessarily the same as in the previous case (around 2.57 in Fig. 5 and around 3.07 in Fig. 3). In addition, Fig. 5 shows that the estimates $\hat{\bar{\omega}}_{i}$ converge to $\omega_{i}^{2}$ asymptotically. Finally, as anticipated after Remark 4 , Figs. 6 and 7 show the effectiveness of (26a)-(26d) and (23e) in practice. Note the convergence of $\beta_{i}$ to a value around 3.07, exactly as in Fig. 3: therefore, convergence occurs to a group model being the average of the actual individuals models.

\section{CONCLUSION}

In order to construct a distributed controller to make heterogeneous agents synchronize to a common trajectory, prior works postulated that a reference model be known to at least some agents. The goal of this work was to lift this assumption,
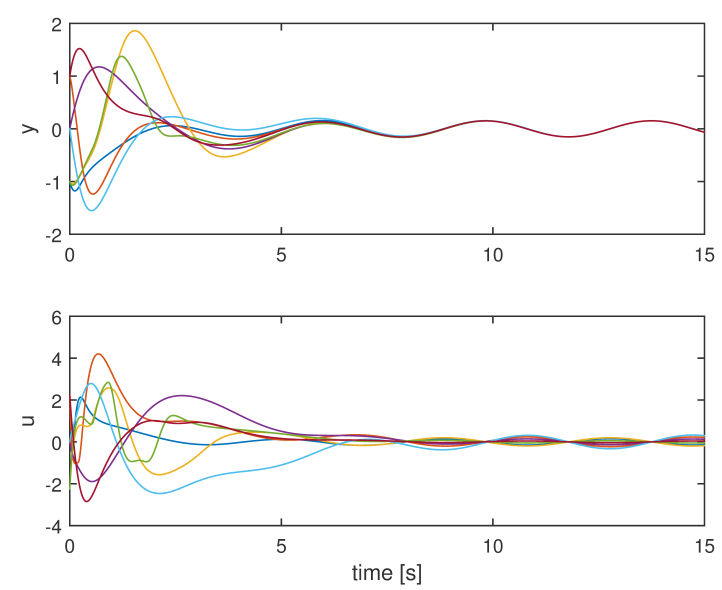

Fig. 4: Unknown $\omega_{i}^{2}$ : synchronizing outputs $y_{i}$ and inputs $u_{i}$.
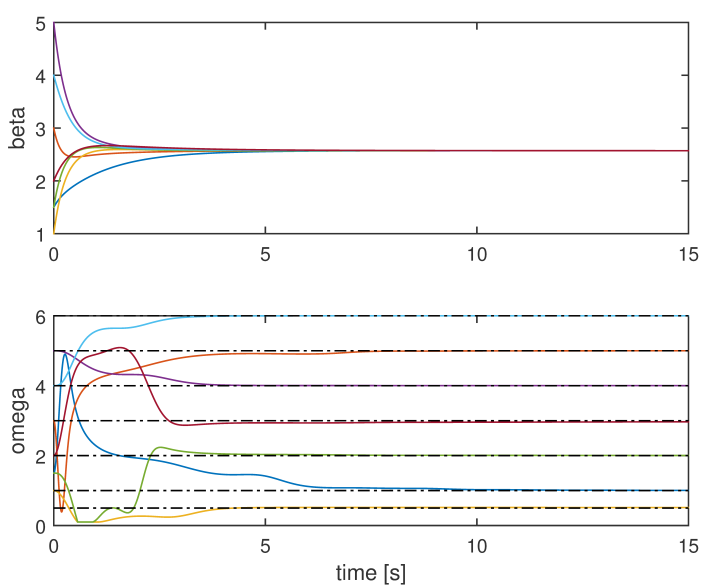

Fig. 5: Unknown $\omega_{i}^{2}$ : convergence of $\beta_{i}$ and of $\hat{\bar{\omega}}_{i}$.

by letting the agents cooperatively learn the parameters of a common model (the group model) that are initially unknown to all agents. We have also shown that even when the agents do not know the parameters of their own dynamics, the group model exists and the agents can cooperatively learn its parameters and synchronize to its dynamics.

Future work can address some of the restrictive assumptions of our results. First, in our learning scheme the observer gain $\kappa$ is common to all systems, in line with the literature on synchronization for heterogeneous systems [13]: a relevant open problem is to design or adapt such gain independently for each agent, similarly to what can be done for homogeneous agents [2]. Second, our work focused on harmonic oscillators as a specific relevant class of (heterogeneous) systems, but we are confident that its ideas can be extended to more general systems. Considering harmonic oscillators has allowed us to derive the structure of the group model analytically and just leave to the agents the task of learning its parameters. Such a structural knowledge, which in the linear case is justified by the fact that the regulation problem typically considers a marginally stable exosystem [31], might be restrictive for more general classes of systems. In future work, it would be 

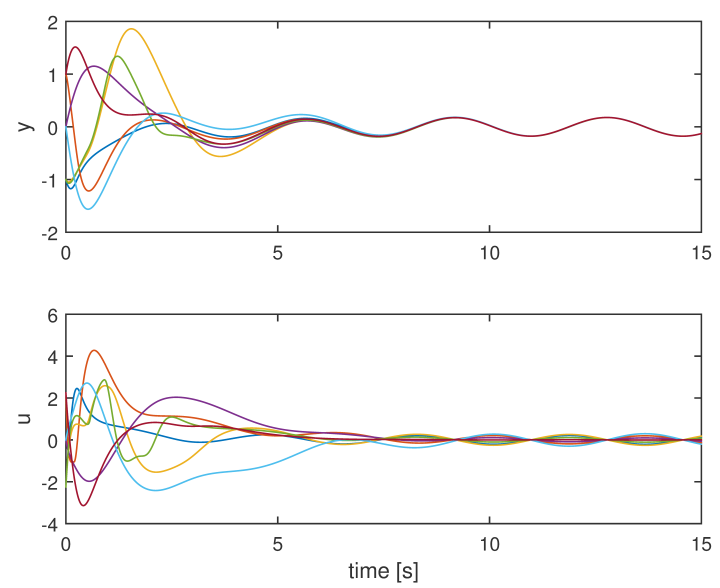

Fig. 6: Unknown $\omega_{i}^{2}$, average model: outputs $y_{i}$ and inputs $u_{i}$.
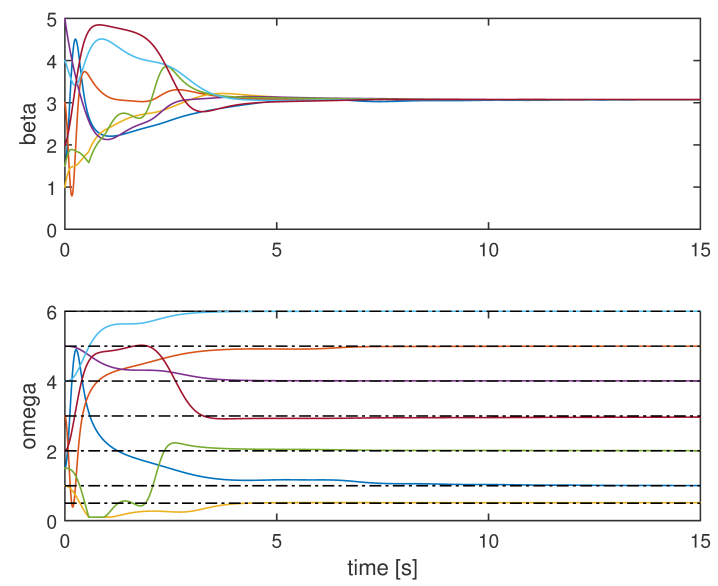

Fig. 7: Unknown $\omega_{i}^{2}$, average model: $\beta_{i}$ and $\hat{\bar{\omega}}_{i}$.

interesting to study whether the group model structure can be learned by the agents themselves, e.g. via neural-network approaches.

\section{REFERENCES}

[1] P. Wieland, R. Sepulchre, and F. Allgöwer, "An internal model principle is necessary and sufficient for linear output synchronization," Automatica, vol. 47, no. 5, pp. 1068-1074, 2011.

[2] L. F. R. Turci, P. De Lellis, E. E. N. Macau, M. Di Bernardo, and M. M. R. Simões, "Adaptive pinning control: A review of the fully decentralized strategy and its extensions," The European Physical Journal Special Topics, vol. 223, no. 13, pp. 2649-2664, 2014.

[3] H. Kim and C. De Persis, "Adaptation and disturbance rejection for output synchronization of incrementally output-feedback passive systems," International Journal of Robust and Nonlinear Control, vol. 27, no. 17, pp. 4071-4088, 2017.

[4] R. Olfati-Saber and R. M. Murray, "Consensus problems in networks of agents with switching topology and time-delays," IEEE Transactions on Automatic Control, vol. 49, no. 9, pp. 1520-1533, 2004.

[5] W. Ren and R. W. Beard, "Consensus seeking in multiagent systems under dynamically changing interaction topologies," IEEE Transactions on Automatic Control, vol. 50, no. 5, pp. 655-661, 2005.

[6] N. Monshizadeh and C. De Persis, "Agreeing in networks: Unmatched disturbances, algebraic constraints and optimality," Automatica, vol. 75, pp. 63-74, 2017.

[7] J. Fax and R. Murray, "Information flow and cooperative control of vehicle formations," IEEE Transactions on Automatic Control, vol. 49, no. 9, pp. 1465-1476, 2004.
[8] G. S. Seyboth, D. V. Dimarogonas, K. H. Johansson, P. Frasca, and F. Allgöwer, "On robust synchronization of heterogeneous linear multiagent systems with static couplings," Automatica, vol. 53, pp. 392-399, 2015.

[9] H. Kim, H. Shim, and J. H. Seo, "Output consensus of heterogeneous uncertain linear multi-agent systems," IEEE Transactions on Automatic Control, vol. 56, no. 1, pp. 200-206, 2011.

[10] Y. Su and J. Huang, "Cooperative output regulation of linear multi-agent systems," IEEE Transactions on Automatic Control, vol. 57, no. 4, pp. 1062-1066, 2012.

[11] M. Lu and L. Liu, "Distributed feedforward approach to cooperative output regulation subject to communication delays and switching networks," IEEE Transactions on Automatic Control, vol. 62, no. 4, pp. 1999-2005, 2017.

[12] G. S. Seyboth, W. Ren, and F. Allgöwer, "Cooperative control of linear multi-agent systems via distributed output regulation and transient synchronization," Automatica, vol. 68, pp. 132-139, 2016.

[13] H. Cai, F. L. Lewis, G. Hu, and J. Huang, "The adaptive distributed observer approach to the cooperative output regulation of linear multiagent systems," Automatica, vol. 75, pp. 299-305, 2017.

[14] W. Chen, X. Li, W. Ren, and C. Wen, "Adaptive consensus of multi-agent systems with unknown identical control directions based on a novel Nussbaum-type function," IEEE Transactions on Automatic Control, vol. 59, no. 7, pp. 1887-1892, 2014.

[15] Y. Su and J. Huang, "Cooperative adaptive output regulation for a class of nonlinear uncertain multi-agent systems with unknown leader," Systems \& Control Letters, vol. 62, no. 6, pp. 461-467, 2013.

[16] Z. Ding and Z. Li, "Distributed adaptive consensus control of nonlinear output-feedback systems on directed graphs," Automatica, vol. 72, pp. $46-52,2016$.

[17] W. Wang, C. Wen, and J. Huang, "Distributed adaptive asymptotically consensus tracking control of nonlinear multi-agent systems with unknown parameters and uncertain disturbances," Automatica, vol. 77, pp. 133-142, 2017.

[18] E. Nuno, R. Ortega, L. Basanez, and D. Hill, "Synchronization of networks of nonidentical Euler-Lagrange systems with uncertain parameters and communication delays," IEEE Transactions on Automatic Control, vol. 56, no. 4, pp. 935-941, 2011.

[19] T. Hatanaka, N. Chopra, M. Fujita, and M. Spong, Passivity-Based Control and Estimation in Networked Robotics. Springer, 2015.

[20] W. Ren, "Synchronization of coupled harmonic oscillators with local interaction," Automatica, vol. 44, no. 12, pp. 3195 - 3200, 2008.

[21] X. Liu and T. Iwasaki, "Design of coupled harmonic oscillators for synchronization and coordination," IEEE Transactions on Automatic Control, vol. 62, no. 8, pp. 3877-3889, 2017.

[22] S. E. Tuna, "Synchronization of harmonic oscillators under restorative coupling with applications in electrical networks," Automatica, vol. 75, pp. 236-243, 2017.

[23] Q. Song, F. Liu, J. Cao, A. Vasilakos, and Y. Tang, "Leader-following synchronization of coupled homogeneous and heterogeneous harmonic oscillators based on relative position measurements," IEEE Transactions on Control of Network Systems, 2018.

[24] H. Zhang, Q. Wu, and J. Ji, "Synchronization of discretely coupled harmonic oscillators using sampled position states only," IEEE Transactions on Automatic Control, vol. 63, no. 11, pp. 3994-3999, 2018.

[25] S. Strogatz, Sync: The emerging Science of spontaneous Order. Hyperion, New York, 2003.

[26] F. Dorfler and F. Bullo, "Synchronization in complex networks of phase oscillators: A survey," Automatica, vol. 50, no. 6, pp. 1539-1564, 2014.

[27] A. Isidori, L. Marconi, and G. Casadei, "Robust output synchronization of a network of heterogeneous nonlinear agents via nonlinear regulation theory," IEEE Transactions on Automatic Control, vol. 59, no. 10, pp. 2680-2691, 2014

[28] Z. Li, Z. Duan, G. Chen, and L. Huang, "Consensus of multiagent systems and synchronization of complex networks: A unified viewpoint," IEEE Transactions on Circuits and Systems I: Regular Papers, vol. 57, no. 1, pp. 213-224, 2010.

[29] P. Ioannou and J. Sun, Robust Adaptive Control. Dover Publications, 2012.

[30] F. Fagnani and P. Frasca, Introduction to Averaging Dynamics over Networks. Springer, 2018

[31] B. A. Francis, "The linear multivariable regulator problem," SIAM Journal on Control and Optimization, vol. 15, no. 3, pp. 486-505, 1977. 